\title{
Review: evidence is incomplete on the benefits and risks of commonly used herbal medicines
}

\author{
Ernst E. The risk-benefit profile of commonly used herbal therapies: ginkgo, St.John's wort, ginseng, echinacea, saw \\ palmetto, and kava. Ann Intern Med 2002 Jan 1;136:42-53.
}

\section{QUESTION: What are the benefits and risks of commonly used herbal medicines?}

\section{Data sources}

Systematic reviews were identified by searching Medline, EMBASE/Excerpta Medica, CISCOM, AMED, and the Cochrane Library (from inception to October 2000), and by contacting experts.

\section{Study selection}

Systematic reviews were selected if they included randomised controlled trials (RCTs) done in humans on ginkgo, St John's wort, ginseng, echinacea, saw palmetto, or kava.

For correspondence:

Dr E Ernst, University of

Exeter, Exeter, Devon,

UK.e.ernst@exeter.ac.uk

A modified version of this

abstract appears in

Evidence-Based

Medicine.

\section{Data extraction}

Data were extracted on the common and manufacturer names and uses of each herbal medicinal product, type and quality of the systematic reviews, sample sizes, and results.

\section{COMMENTARY}

In 1998, Eisenburg et al reported that use of herbal treatments had increased $380 \%$ from 1990 to 1997 and that 1 in 5 people who were taking prescription drugs were also taking herbs, high dose vitamins, or both. ${ }^{1}$ The well done review by Ernst assessed the efficacy and safety of herbal treatments. The quality of included studies was evaluated, and weak trials were identified. Ernst notes that publication bias was a factor in the evaluation of ginkgo for cognitive function (excluding dementia), and echinacea for the treatment and prevention of upper respiratory tract infections. St John's wort appears to be efficacious for mild to moderate depression despite methodological flaws in earlier studies.

The field of herbal treatments is ever changing and Ernst's challenge that physicians "acquire sufficient knowledge in this area to advise their patients responsibly" is a challenge for all healthcare providers. Furthermore, most patients do not disclose their use of herbal products or complementary treatments to their healthcare providers. ${ }^{1}$ New data on the safety of herbal products continue to be reported, including a recent warning that approximately 25 reports of liver toxicity in Germany and Switzerland have been associated with kava use. ${ }^{2}$

Interactions between herbal treatments and other drugs are a serious concern. Also, because of the low quality of existing studies, many interactions may not be recognised. Ernst highlights other safety issues, including the lack of quality control and standardisation of herbal treatments that pose risks from contamination, underdosing, or both. Rigorous studies are needed to identify adverse effects and inherent dangers associated with the combined use of prescription drugs and herbal products. If one is to use herbal products, only those brands found to be effective in clinical trials should be used. Herbal remedies need to be further evaluated before practitioners can adequately assess risk-benefit ratios

Diane Wardell, RNC, PhD Associate Professor School of Nursing, University of Texas Houston Houston, Texas, USA

1 Eisenberg DM, Davis RB, Ettner SL, et al. Trends in alternative medicine use in the United States, 1990-1997: results of follow-up national survey. JAMA 1998;280:1569-75.

2 Food and Drug Administration MEDWATCH. The FDA Safety Information and Adverse Event Reporting Program. 2001 Safety Information Summaries. Washington, DC: Food and Drug Administration (last updated 21 Dec 2001). www.fda.gov/medwatch/safety/ 2001/safety01.htm\#kava

\section{Main results}

2 reviews (of adequate and good quality) of ginkgo showed positive effects on memory impairment, although these results are questionable because of the possibility of publication bias. 2 reviews (of adequate to good quality) of ginkgo showed beneficial effects in dementia. 1 adequate quality review of ginkgo for tinnitus also had questionable results because of the lack of rigorous trials. 1 good quality review of ginkgo for intermittent claudication showed that ginkgo led to moderate and probably clinically relevant increases in pain free walking distances. Ginkgo's adverse effects were usually mild, transient, and reversible.

Several good quality reviews showed that St John's wort was more effective than placebo for mild to moderate depression. 1 meta-analysis of 14 RCTs showed that St John's wort was more effective than placebo (relative benefit $1.9,95 \%$ CI 1.2 to 2.8 ) and similar to tricyclic antidepressants (relative benefit 1.2, CI 1.0 to 1.4) for depressive disorders. However, St John's wort may interact with several prescription drugs (eg, anticoagulants, oral contraceptives, and antiviral agents), which may have clinically serious consequences.

1 adequate quality review showed that ginseng was not beneficial as a sedative, hypnotic, demulcent, aphrodisiac, antidepressant, or diuretic agent. 1 good quality review showed that echinacea may be effective in preventing and treating upper respiratory tract infections, but trial data are not rigorous or conclusive. 1 good quality review showed that saw palmetto might reduce symptoms of benign prostatic hyperplasia (eg, nocturia and peak urinary flow) in the short term. Adverse effects of saw palmetto were rare and mild. 1 adequate quality review showed that kava might have short term effectiveness in treating anxiety; serious adverse effects of kava were rare.

\section{Conclusions}

Evidence suggests that St John's wort may be effective in mild to moderate depression, although the effects may be overestimated. Data from weak studies suggest that echinacea may be effective in preventing and treating upper respiratory tract infections. Saw palmetto may have short term effectiveness in benign prostatic hyperplasia, and kava may have short term effectiveness in anxiety treatment. Data on ginkgo show positive effects for dementia and intermittent claudication, but are questionable for cognitive function and tinnitus. Ginseng is not beneficial for a variety of conditions. Overall, evidence is incomplete on the benefits and risks of these commonly used herbal medicines. 